\title{
Connecting Beyond the Research Community: IceCube Education, Outreach, and Communication Efforts
}

The IceCube Collaboration ${ }^{\dagger}$, and E. Bechtol ${ }^{\ddagger}$, S. Bravo ${ }^{\ddagger}$, J. DeMerit ${ }^{\ddagger}$, E. Feitlinger ${ }^{\ddagger}$

${ }^{\dagger}$ http://icecube.wisc.edu/collaboration/authors/icrc17_icecube

* Wisconsin IceCube Particle Astrophysics Center (WIPAC), University of Wisconsin-Madison, Madison, WI 53703, USA

E-mail: silvia.bravo@icecube.wisc.edu, james.madsen@uwrf.edu

As an international organization, the IceCube Neutrino Observatory has 48 collaborating institutions, each of which carries out efforts to engage and inform the public. Examples of activities include one-time talks and posts on social media, support for local science and technology programs, and development of visualization tools and inexpensive detectors for student use. In this paper, we describe four successful examples of centrally supported IceCube education, outreach, and communication efforts to reach beyond the research community. First, we give an overview of the field deployments to the South Pole for high school teachers that we have provided in partnership with the PolarTREC program, supported by the US National Science Foundation. This program enables high school teachers to join the IceCube team and experience firsthand the challenges of doing cutting-edge research in the extreme Antarctic environment. Next we describe the IceCube Masterclass, a day-long event held at multiple IceCube Collaboration campuses for motivated high school students. It was offered for the fourth time in spring 2017. After that, we describe science news articles, short summaries of IceCube results and activities, regularly posted on the IceCube webpage and promoted via social media. The last example we discuss are IceCube videos, some produced in conjunction with prominent partners such as TED-Ed, which have been seen by more than two hundred thousand viewers. A few of the most noteworthy IceCube science results have been followed up with in-house-produced videos. For example, a video on IceCube's sterile neutrino results has received more than ten thousand views. We will provide metrics that describe the reach of these activities along with early efforts to assess the impact of IceCube's education, outreach, and communication activities.

Corresponding authors: S. Bravo ${ }^{1}$ and J. Madsen ${ }^{2 *}$, Presenter: E. Feitlinger ${ }^{1}$

${ }^{1}$ Wisconsin IceCube Particle Astrophysics Center (WIPAC), University of Wisconsin-Madison, Madison, WI 53703, USA

${ }^{2}$ Dept. of Physics, University of Wisconsin, River Falls, WI 54022, USA

35th International Cosmic Ray Conference - ICRC2017

10-20 July, 2017

Bexco, Busan, Korea

${ }^{*}$ Speaker. 


\section{Introduction}

The IceCube Neutrino Observatory consists of a cubic-kilometer neutrino detector installed in the ice at the geographic South Pole, between depths of $1450 \mathrm{~m}$ and $2450 \mathrm{~m}$, and a square-kilometer surface array known as IceTop [1]. The breadth and depth of the facility's science reach is indicated in Fig. 1 and by the number and variety of contributions submitted for ICRC2017 on behalf of the IceCube Collaboration. Science topics of particular relevance to the ICRC include measuring cosmic ray spectra, composition, and anisotropy; characterizing the astrophysical neutrino flux; searching for neutrino sources; and looking beyond the standard model physics for dark matter, sterile neutrinos, and other exotic particles.

The wide range of IceCube topics, many of which are unfamiliar to all but expert audiences, makes producing effective education, outreach, and communication both challenging and rewarding. Conveying what the IceCube project is doing and why, and telling the stories of the people who make it possible, is a central role of the IceCube project office. In addition, many IceCube Collaboration members also take on this important task, motivated by their enthusiasm for science and to meet funding agency broader impact requirements. Their efforts include giving talks to schools and community groups, hosting local science open houses, participating in associated activities arranged by others, such as the International Cosmic Day [2] or a museum exhibit on detector technology [3], and mentoring high school students. In addition, several IceCube collaborators are working on virtual reality displays of varying degrees of sophistication [4], designing and producing apps that utilize cell phones to capture cosmic ray events [5], and even developing inexpensive stand-alone detectors simple enough for classroom use [6].

This paper will focus on four successful examples of collaboration-wide education, outreach, and communication efforts. The goal is to show how we leverage central resources to reach interested audiences, including those outside the research community. Our efforts involve providing very intensive experiences for a few select K-12 teachers, hosting masterclasses for hundreds of high school students, writing articles on science news updates for our website, regularly reaching thousands, and

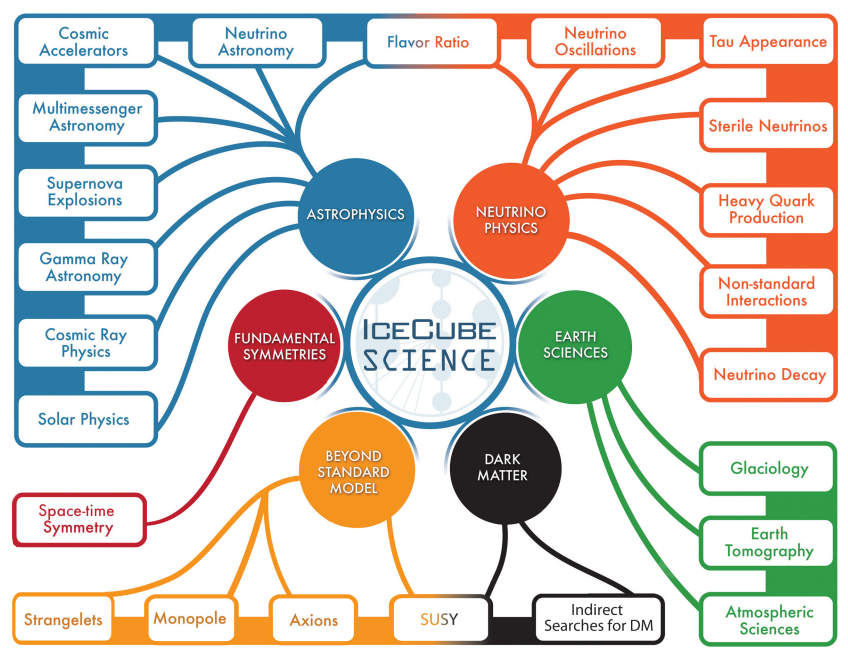

Figure 1: Science topics of the IceCube Neutrino Observatory. producing short informational science videos, with 1,000s to 100,000s of views. A brief discussion at the end will describe metrics we are developing to assess the reach and impact of these efforts. 


\section{Collaboration-wide Examples}

The IceCube project maintenance and operations efforts are led by the Wisconsin IceCube Particle Astrophysics Center (WIPAC) at the University of Wisconsin. WIPAC maintains the IceCube website, Facebook and Twitter accounts, and a YouTube channel [7], as digital resources to reach broader audiences. Funding from the maintenance and operation grant is leveraged with other sources and partnerships to support collaboration-wide education, outreach, and communication activities.

\subsection{PolarTREC: Field Deployments for Teachers}

The NSF-supported PolarTREC program pairs educators, primarily precollege teachers, with polar researchers [8]. The aim of the project is to improve the understanding of the polar regions and the research that takes place there. Teachers are integrated into a research team, participate in a field deployment, and use their skills in translating often arcane, abstract science into accessible language and activities for the classroom. The IceCube project has had five PolarTREC teachers deploy to the South Pole, with the sixth scheduled for the upcoming 2017-18 season ${ }^{1}$ (expedition history shown in Fig. 2). We have had success both with teachers we knew prior to selection as well as with those we chose based solely on written application materials and phone interviews.

The training of a PolarTREC teacher

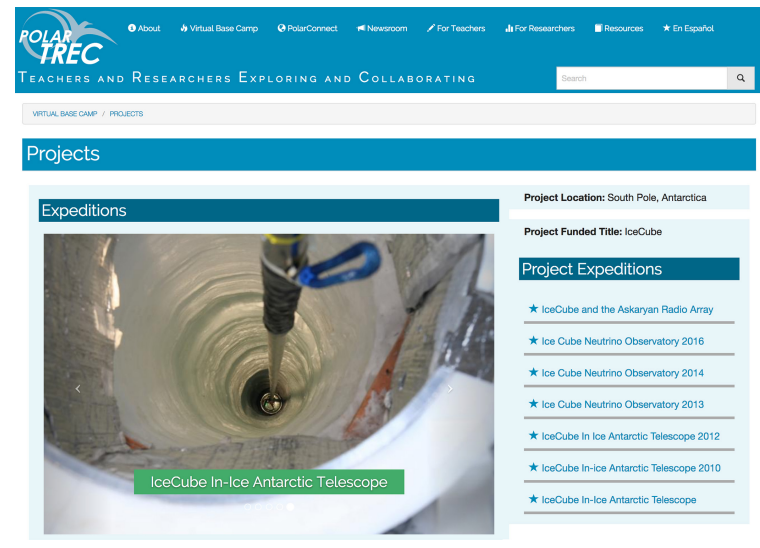

Figure 2: IceCube project history on PolarTREC website [9].

activities to maximize the impact of their experience.

Working with the PolarTREC program enriches IceCube, the teacher, and their communities. The teachers are highly motivated, excited, and anxious to convey what they are learning. They bring their unique perspectives and skills, and they develop materials that document their experiences, including videos, journals, and photos that are posted in real time and then archived on their PolarTREC web page. In addition, their deployments provide a pulse of activity that captivates

\footnotetext{
${ }^{1}$ AMANDA, the proof-in-concept project prior to IceCube, hosted three additional teachers [10].
} 
local and national media and brings awareness of IceCube research to new and diverse audiences. For example, Puerto Rican educator Armando Caussade's deployment received extensive coverage throughout Puerto Rico, including print and broadcast media. He posted all of his materials on his PolarTREC web page [11], in both Spanish and English, and published a book based on his trip, "A Puerto Rican in the South Pole" [12]. Other video resources produced by IceCube PolarTREC teachers are described in $\operatorname{Sec} 2.4$.

\subsection{IceCube Masterclass: Intensive Day-long Experiences for Students}

In 2014, IceCube launched the IceCube Masterclass [13], an educational research-based program for high school students that aims to excite the next-generation of researchers in astrophysics. The IceCube Masterclass program was inspired by the International Masterclasses program, started in 2005 by the International Particle Physics Outreach Group, and supported in the U.S. by QuarkNet [14].

During a masterclass, high school students, and sometimes accompanying teachers, join an IceCube institute for a day to engage in real research and experience an authentic and international scientific environment. Students are guided through one IceCube analysis, either the discovery of astrophysical neutrinos or the measurement of the cosmic ray spectrum [15]. The focus is to illustrate how astrophysics is done by reproducing an important IceCube paper, condensing the steps to allow the exercise to be completed in a few hours and avoiding the technicalities of handling large amounts of data.

The first IceCube Masterclass hosted around 100 students at five institutions in the U.S. and Europe. The fourth edition took place in March 2017 with over 200 students in Belgium, Denmark, Germany, and the US, at a total of 14 institutions. The activities were designed for students in their last two years before college, although a few younger students who were especially interested in astrophysics have also participated.

Each year, the program is evaluated using pre- and post-program surveys containing a mix of open-ended, multiple-choice, and rating questions [16]. Students showed gains in knowledge and skills, and they left feeling more excited about science and physics. They were asked to assess each activity of the day on a scale of 1-5. The analysis activity ranked highest, with an average score per institution above 4 .

\subsection{Detector and Research News: a Feed for the IceCube Website and Social Networks}

The IceCube website publishes on average two news articles every week. These include weekly updates about activities at the South Pole, summaries of every research paper published by the IceCube Collaboration, features on detector operations or outreach activities, and press releases and announcements about outstanding results and awards honoring the IceCube Neutrino Observatory and/or the international team behind it. These news items are the main dynamic content of the website and also feed IceCube Facebook and Twitter accounts.

These news posts consistently account for around $10 \%$ of the page views on the IceCube website, but their impact is much larger in terms of audience reach and overall IceCube awareness. Although only $50 \%$ of these articles get over 1,000 readers on the website, and less than $20 \%$ reach 3,000 readers or more, they trigger a shower of communication that can amplify our audience by a factor of 10 or more. 


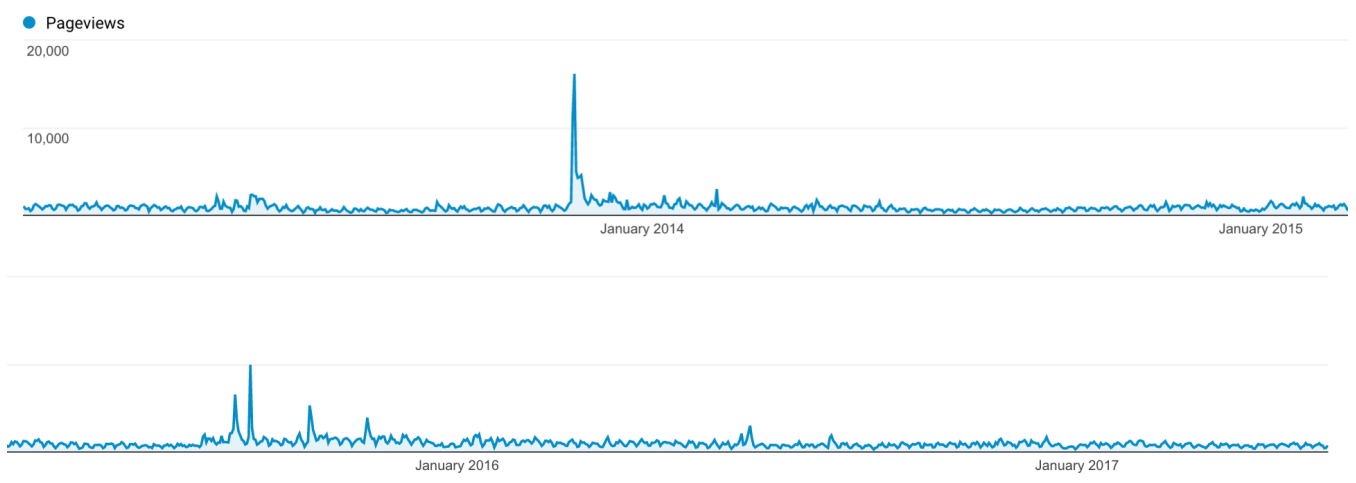

Figure 3: Daily page views of IceCube's website since 2013. The largest peak corresponds to the publication in Science of the discovery of astrophysical neutrinos.

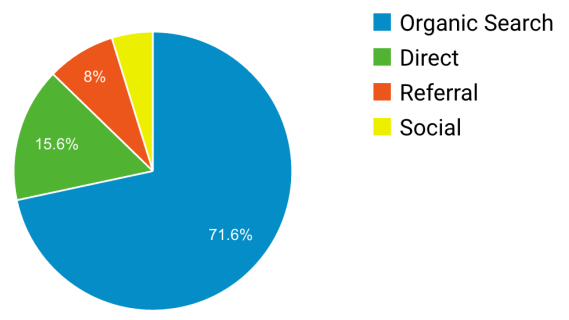

Figure 4: Top channels bringing traffic to IceCube's website.
On an average day, over 400 users visit the IceCube website, and $25 \%$ of those are returning visitors. This number varies considerably, with peaks that correspond to the publication of articles (see Fig. 3). The most successful news article-the press release about the discovery of astrophysical neutrinos published in Science in November 2013 [17]—brought in over 10,000 users in less than 48 hours. However, a closer look at the data shows that although this peak was triggered by the release, not all users came to our website to learn about the discovery. Only about half

$(4,702)$ of the 10,000 users read that news during those two days (currently the news has over 7,400 views). So, thousands of visits were triggered by the news in the media, or on social networks, and came to the website to learn more about IceCube. This effect is confirmed by comparing the page views of the first five months of 2017 with five months around the Science paper. The percentage of views of IceCube news was in both cases around $11 \%$, i.e. the effect of the discovery was diluted when taking into account the views over a few months. However, the visits to the home page increased by $30 \%$, with a huge peak around the IceCube discovery (visits to the home page usually are in the few hundreds per day, and were over 5,000 the day of the release). At the same time, the percentage of visits to static sections, such as science, outreach or the collaboration, decreased by $50 \%$ or more. The readers of content related to the South Pole remained stable at around $15 \%$, showing the appeal of Antarctica for all audiences.

The increase in visits to the home page can be easily explained if we look at the origin of website users, who mostly reach IceCube content as a result of an organic search, i.e., after looking for content by entering key words in a browser search engine (see Fig. 4 for distribution).

A similar cascade effect is observed on IceCube social networks. On Facebook, with just over 8,000 followers, posts of research news that have one or two thousand readers, can easily reach 5,000 to 10,000 followers, and only a few of them (typically around a 5\%) will actually click on the news link. This amplifying effect can also be observed in the patterns of new likes on Twitter 
that mimic the impact of IceCube news on this social network. IceCube's Twitter feed currently has around 6,200 followers. Fig. 5 shows recent Twitter activity.

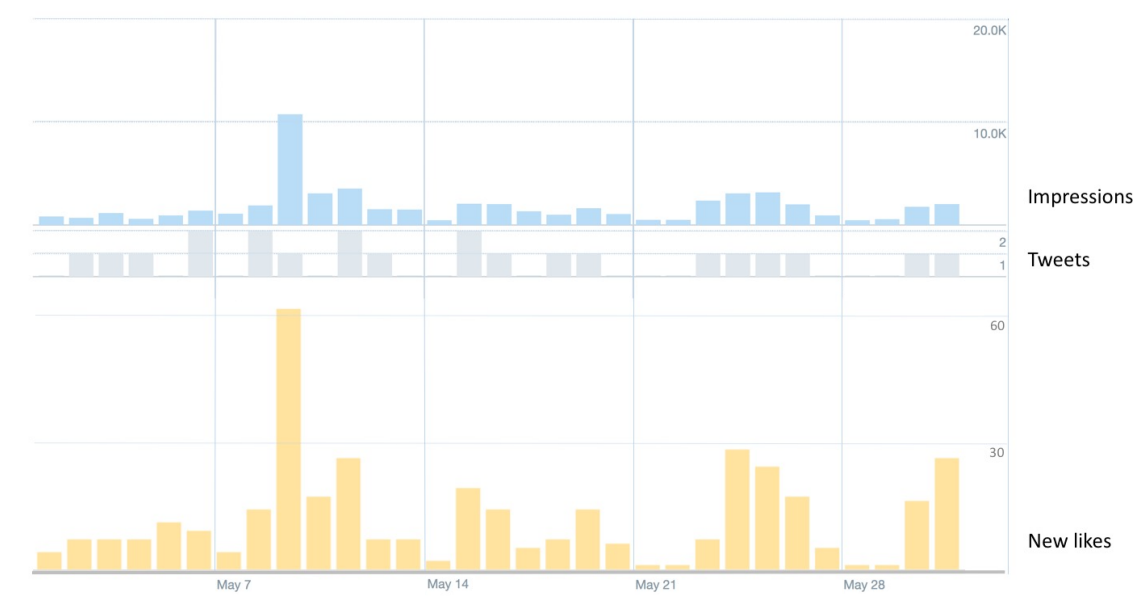

Figure 5: Twitter impressions in May 2017 (blue) and number of tweets (grey). The yellow graph shows the number of new likes for the same period.

\subsection{IceCube Videos: In-house and Partner Productions}

In the era of mobile devices and multimedia content, it is well known that animated graphics and video productions can engage much larger audiences than text-based content and static images. However, production of multimedia resources takes more human and capital resources. IceCube uses a strategy that leverages internal resources by combining in-house and partner productions. We have worked with the TED-Ed portal (Fig. 6), Spanish filmmaker Javier Diez, the Milwaukee Public Museum, and the American Physical Society to produce several videos introducing IceCube to the public. As of May 2017, they have reached over 210,000 views [18, 19, 20, 21].

Several of the PolarTREC teachers have also pro-

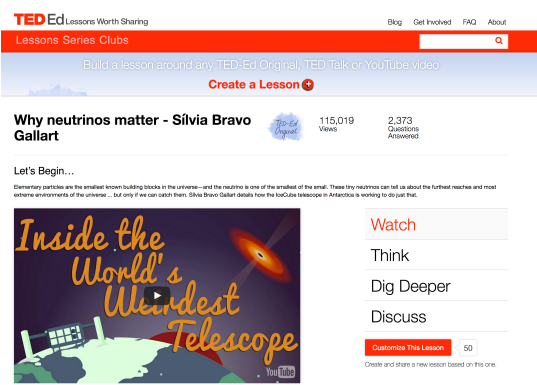

Figure 6: TED-Ed lesson about IceCube, including a video and questions for using the lesson in formal and informal learning. duced videos prior to and during their visits to the IceCube Neutrino Observatory at the South Pole. During her deployment in January 2017, teacher Kate Miller published a video touring the South Pole station that currently has almost 175,000 views. In 2009, another PolarTREC teacher, Casey O'Hara, produced a novel stop-motion animation about IceCube that continues to be popular, and has around 23,500 views $[22,23]$.

During the last year, IceCube has also internally launched the regular production of short videos, including a series featuring IceCube research. The first of these videos, in conjunction with a press release about a search for sterile neutrinos, has accumulated over 14,000 views on the IceCube YouTube channel, and several thousand more viewers on the websites of Wall Street Journal and Space.com [24]. 


\section{Education, Outreach and Communication Metrics}

We are currently working on a new and broader evaluation framework to assess the impact of IceCube activities, with a special focus on measuring the accomplishment of main goals related to target audiences. The aim is to move beyond only measuring and reporting the number of web users, Facebook and Twitter followers, video viewers, or participants at outreach programs and events. This framework is mostly implemented in the evaluation of the IceCube Masterclass outreach program, with the long-term goal of measuring the impact this intensive activity (about eight hours of contact per participant) has on fostering research careers.

For other IceCube activities, we are just starting to move beyond recording the number of our participants and gather demographic information on our audiences. A common goal of IceCube communications and outreach programs is to reach and serve target communities, such as groups underrepresented in science careers, K-12 students and teachers, science aficionados, and the broader IceCube community. Online platforms such as websites and social networks have analytic tools to parse simple metrics, for example, gender and home country or language.

Preliminary studies about gender reach show a predominantly male audience, with only $22 \%$ of IceCube followers being women on Twitter, $27 \%$ on Facebook, and $32 \%$ on the IceCube website. Gender metrics are from Google Analytics summaries.

Regarding origin, IceCube's audience is primarily from English-speaking locations. English is the language used on all IceCube official communication channels, and English-speaking countries, including the US, Canada, Australia, and the UK, host more than $50 \%$ of the IceCube institutions. However, small efforts towards multilingualism are already showing that this audience can be diversified. The minisite of the IceCube Masterclass published the same content in three languages: English, German, and Spanish. On this website, Germany is second in the number of users - it is the fourth on the main website. The list of the top 10 countries in the masterclass website also includes Spain and Mexico, countries that do not show up in the top 10 countries for the main website (see Table 1 for reference).

\begin{tabular}{cc}
\multicolumn{2}{c}{ IceCube } \\
\hline Main \\
website & $\begin{array}{c}\text { Masterclass } \\
\text { minisite }\end{array}$ \\
\hline US & US \\
UK & Germany \\
India & UK \\
Germany & Russia \\
Canada & Spain \\
Australia & India \\
Russia & Belgium \\
Italy & Italy \\
Japan & Mexico \\
France & Japan
\end{tabular}

Table 1: List of the top 10 countries for the main website and the masterclass minisite.

On a wider scope, IceCube has not yet implemented a collaboration-wide assessment model. This would allow the aggregation of data from the vast number of programs and activities run by the education and outreach office at UW-Madison, headquarters of the IceCube Neutrino Observatory, together with local programs at each IceCube institution (48 in 12 countries). A design for such a model is currently under development. It will include the identification of the relevant metrics based on recent research on the assessment of broader impacts and the creation of a flexible tool that can be used by IceCube collaborators around the world.

\section{Summary and Outlook}

The impact of science extends beyond the knowledge and technology produced. Increasingly, research funders are requiring scientists to engage in education, outreach, and more formal efforts 
to communicate beyond the traditional research community. The expectations and investments in these activities scale with the size of the project. Four examples of IceCube's ongoing, centrally supported education, outreach, and communication efforts were described. These activities are providing ways to connect to IceCube science and personnel on a variety of levels and formats. They range from research experiences including field deployments for teachers and masterclasses for high school students that provide intensive, face-to-face contacts to digital resources like weekly web news and video content. We have good data on the number of people who consume media or participate in events. The next step is to learn more about who these people are to ensure that the desired audiences are being reached, and that activities are having the desired impact.

\section{References}

[1] IceCube Collaboration, M. G. Aartsen et al., JINST 12 (2017) P03012

[2] International Cosmic Day: https://icd.desy.de

[3] IceCube exhibit at Gustavianum Museum in Uppsala: http://www.gustavianum.uu.se/gustavianumeng/exhibitions/temporary-exhibitions/aspiring-to-precision/the-exhibition/iceCube

[4] Polar Virtual Reality project at Wisconsin Institutes of Discovery: https://pvre.discovery.wisc.edu

[5] DECO: https://wipac.wisc.edu/deco/home

[6] S. Axani, J. Conrad and C. Kirby, arXiv:1606.01196 [physics.ed-ph]

[7] http://icecube.wisc.edu; https://twitter.com/uw_icecube; https://twitter.com/UWastrophysics; https://www.youtube.com/channel/UCqhypTo6SWmi5bbVBmUf9NA

[8] PolarTREC: www.polartrec.com

[9] IceCube PolarTREC expeditions: https://www.polartrec.com/projects/icecube-project

[10] See Muhs, Pettersson, Petula, http://tea.armadaproject.org/tea_meetteachers.html\#antarctic

[11] Armando Caussade's PolarTREC page: https://www.polartrec.com/member/armando-caussade

[12] "A Puerto Rican in the South Pole": https://www.polartrec.com/resources/article/a-puerto-rican-in-the-south-pole

[13] IceCube masterclass: https://masterclass.icecube.wisc.edu

[14] International Particle Physics masterclass: http://www.physicsmasterclasses.org

[15] IceCube Collaboration, PoS ( ICRC2015) 576 (2016).

[16] "Searching for Cosmic Neutrinos in IceCube, an IceCube Masterclass research-based activity," Physics Teacher, in press.

[17] IceCube Collaboration, M. G. Aartsen et al., Science 342 (2013) 1242856

[18] "Why Neutrinos Matter," http://ed.ted.com/lessons/why-neutrinos-matter-silvia-bravo-gallart

[19] "Neutrino, Measuring the Unexpected," https://vimeo.com/114361247

[20] "Chasing the Ghost Particle," https://wipac.wisc.edu/ghostparticle

[21] “IceCube Explained," https://www.youtube.com/watch?v=xUit5_B9k-U

[22] "Tour of South Pole Station," https://www.youtube.com/watch?v=vrPiVT23MhA

[23] "IceCube - Animated Overview," https://www.youtube.com/watch?v=aMnGWqoDaAA

[24] "The Search for Sterile Neutrinos in IceCube," https://www.youtube.com/watch?v=tlMoFsRlrew 